\title{
SIMULACIÓN DEL PROCESO DE CARBONATACIÓN DEL HORMIGÓN EN TÚNELES URBANOS
}

\author{
$\underline{\text { L. López-de Abajo }}^{1 *}$, J. C. Gálvez ${ }^{1}$, M. G. Alberti ${ }^{1}$ \\ *Autor de Contacto: lucia.lopezdeabajo@upm.es \\ ${ }^{1}$ Departamento de Ingeniería Civil: Construcción, E.T.S de Ingenieros de Caminos, Canales y Puertos, Universidad \\ Politécnica de Madrid. C/ Profesor Aranguren s/n, 28040, Madrid, España.
}

\section{RESUMEN}

El objetivo del presente trabajo es determinar, mediante el empleo de un modelo numérico, el proceso de la carbonatación en el hormigón que se encuentra sometido a altas concentraciones de gases, como las existentes en los túneles urbanos. De esta manera, se obtiene una herramienta que, además de permitir conocer el estado de deterioro actual del material, posibilita el llegar a predecir su evolución en el tiempo. Esta funcionalidad es de gran importancia para el mantenimiento y reparación de estas infraestructuras, dado que hace posible el diagnóstico de sus patologías sin afectar al tráfico. Para ello, se está realizando la adaptación de un modelo ya existente a los condicionantes propios de un túnel urbano y su calibración mediante una campaña experimental.

Palabras clave: carbonatación; dióxido de carbono; hormigón; simulación numérica; túnel urbano.

\section{ABSTRACT}


The aim of the present work is to assess the extent of the carbonation process in the concrete that constitutes the structure of urban road tunnels. This assessment is made by simulation using a numerical model. Thus, a powerful tool is developed, as it not only determines the real damage level of the concrete, but it can also predict its deterioration in the future. This feature is particularly relevant for the maintenance and reparation of these kind of infrastructures, as it allows to diagnose the damage of the concrete without interrupting the traffic flow. To this effect, the adaptation of an existing model to the peculiarities of an urban tunnel is being carried out, and also its calibration with an experimental campaign.

Key words: carbonation; carbon dioxide; concrete; numerical simulation; urban tunnel.

\section{INTRODUCCIÓN. EL DETERIORO DE LOS TÚNELES URBANOS}

Los túneles, por tratarse de estructuras enterradas y por tanto, en contacto directo con el terreno, presentan unas patologías particulares que las diferencian de otras estructuras (Li et al., 2018). Dentro de los daños que se pueden encontrar en un túnel, se puede diferenciar entre aquellos deterioros que afectan a su equipamiento (daños en el sistema de ventilación, megafonía, etc. (Llopis Serrano, 2012)) o aquellos que están relacionados con el propio túnel. En lo que respecta a estos últimos, a su vez, se encuentran los deterioros que afectan a la explotación, es decir, a los vehículos y a los usuarios (como pueden ser excesos de mortero en la losa superior que puedan precipitarse a la calzada) o aquellos que afectan al túnel como estructura. Dentro de estos deterioros se distinguen las patologías que afectan a la resistencia de la estructura (aquellas que afectan a su capacidad de resistir las cargas a las que se ve expuesto) o las que afectan a su durabilidad (cómo responde la estructura ante el ambiente en el que se encuentra) (Ministerio de Fomento, 2010). Teniendo siempre en cuenta que un deterioro relacionado con la durabilidad, si se mantiene en el tiempo y evoluciona desfavorablemente, puede terminar afectando a la resistencia de la estructura (Ministerio de Fomento, 2012).

El presente trabajo se centra en los deterioros de durabilidad que afectan al hormigón armado que conforma la estructura de los túneles urbanos. Y, entre todos los deterioros que pueden dañar este material (lixiviación, ataque por sulfatos, hielo-deshielo, ataque por cloruros, árido-alcali, etc. (Galan et al., 2019)), en la neutralización por exposición a gases, y concretamente, en la carbonatación del hormigón.

La carbonatación es uno de los mecanismos de deterioro más habituales del hormigón armado. Tiene lugar cuando se produce la absorción de $\mathrm{CO}_{2}$ en presencia de humedad. Este $\mathrm{CO}_{2}$ absorbido se disuelve en la fase acuosa de los poros y reacciona con el calcio procedente de los compuestos de hidratación del cemento (portlandita, gel CSH, aluminatos y sulfoaluminatos) (Richardson, 2002). Debido a esta reacción se genera $\mathrm{CaCO}_{3}$ y agua según la fórmula (1), que se encuentra particularizada para la carbonatación de la portlandita.

$$
\mathrm{Ca}(\mathrm{OH})_{2}+\mathrm{CO}_{2} \rightarrow \mathrm{CaCO}_{3}+\mathrm{H}_{2} \mathrm{O}
$$

Si bien produce una serie de efectos positivos para la matriz del hormigón, como pueden ser la reducción de porosidad (Tongaria, et al., 2018), el posible aumento de resistencia a tracción y compresión (Galán, 2011) o la reducción de su permeabilidad (Phung et al., 2016); este proceso puede comprometer la durabilidad del acero de las armaduras del hormigón, dado que se produce 
un descenso de su pH de 12,6 (hormigón sano) a 8,3 y es conocido que las armaduras pierden su capa pasiva protectora cuando el $\mathrm{pH}$ de su entorno es inferior a 9 y puede producirse su corrosión (Richardson, 2002).

Cabe destacar que la carbonatación se trata de un fenómeno complejo, dado que en ella intervienen una gran cantidad de parámetros, tanto de composición del hormigón, como de ejecución y de ambiente de exposición, que se encuentran relacionados entre sí (López-de Abajo et al., 2020; Singh and Singh, 2016).

Este fenómeno se está estudiando en unos túneles urbanos de gran relevancia para la ciudad de Madrid: los túneles de Calle 30 (López-de Abajo et al., 2020) (cuya denominación era M-30 antes de la cesión al Ayuntamiento). Madrid cuenta con 3,5 millones de habitantes y su área metropolitana con unos 6 millones (Perez-Prada and Monzon, 2014), lo que la convierte en la mayor ciudad de España (Núñez-Alonso et al. 2019). La Calle 30 es uno de los anillos de circunvalación que rodean la ciudad de Madrid, en concreto el más interior, con una IMD aproximada de $200.000 \mathrm{veh} /$ día y una longitud total de $32 \mathrm{~km}$ (Perez-Prada and Monzon, 2017). Esta infraestructura se construyó entre los años 1970 y 1990 y entre los años 2004 y 2007 se 1 levó a cabo el soterramiento de su zona sur, fecha en la que se construyeron los túneles actuales, que tienen una longitud de 8,8km (Perez-Prada and Monzon, 2017).

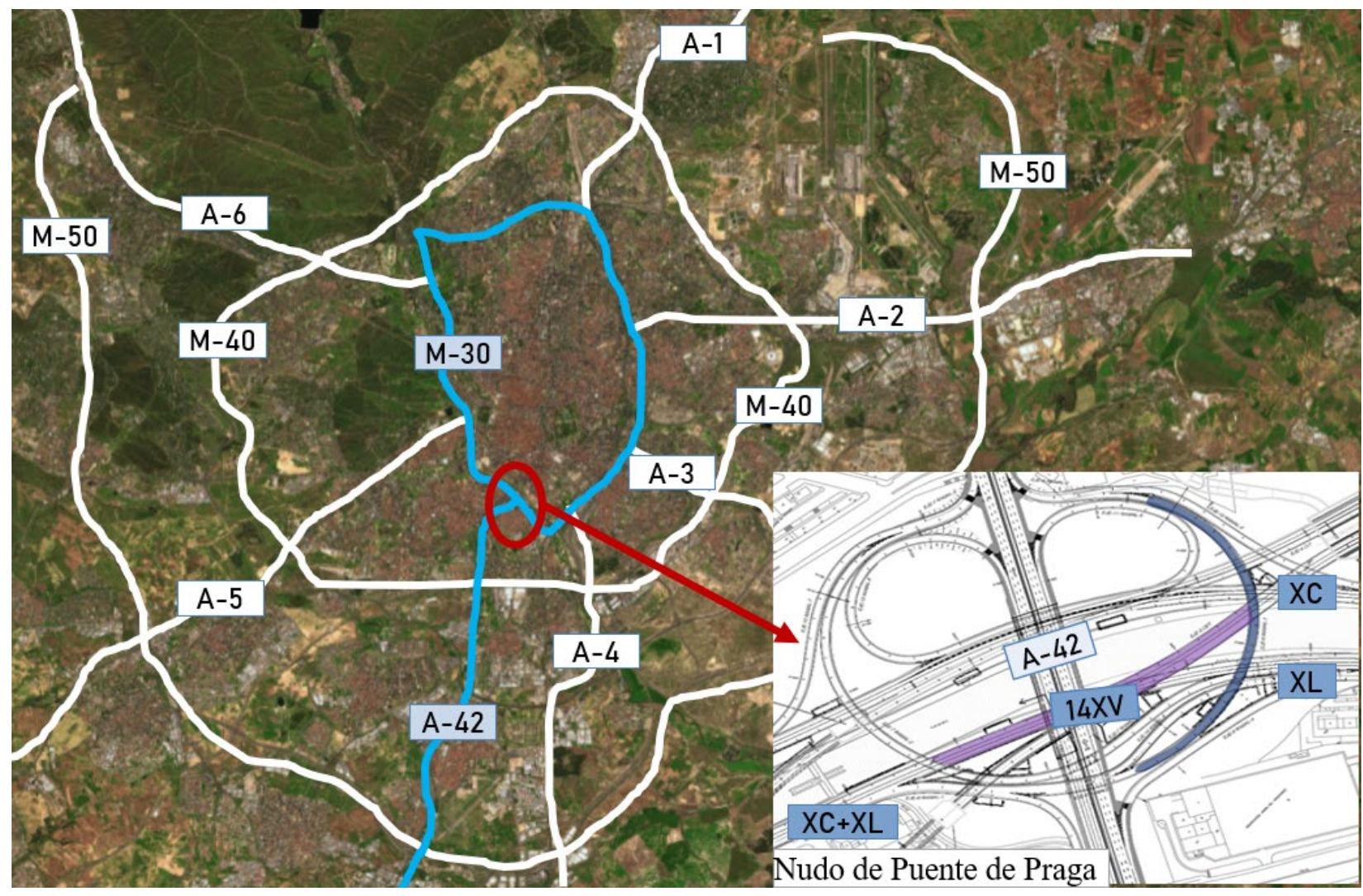

Figura 1. Situación de la galería de ventilación $14 \mathrm{XV}$, Nudo de Puente de Praga y principales vías de circulación.

Concretamente, la zona de estudio se encuentra en el Nudo del Puente de Praga (Figura 1), que materializa el cruce de la Calle 30 con la carretera A-42. En esta zona se produce la particularidad de que las dos calzadas que circulan hacia el sur (XC y XL) se encuentran superpuestas y entre 
ellas se localiza una galería de ventilación (14XV) que absorbe los gases producidos por los vehículos que circulan por la calzada y los expulsa hacia el exterior.

Esta galería tiene unas dimensiones aproximadas de $345 \mathrm{~m}$ de longitud y $15 \mathrm{~m}$ de anchura media y en la Figura 2 (izquierda) se muestra su sección transversal. La zona central de la galería se corresponde con la zona de circulación del aire sucio extraído de la calzada y dispone de dos conducciones de inyección de aire limpio a ambos lados. Se muestra en la Figura 2 (derecha) una imagen tomada en el interior de la galería, en la que se aprecian las mencionadas conducciones de aire limpio.
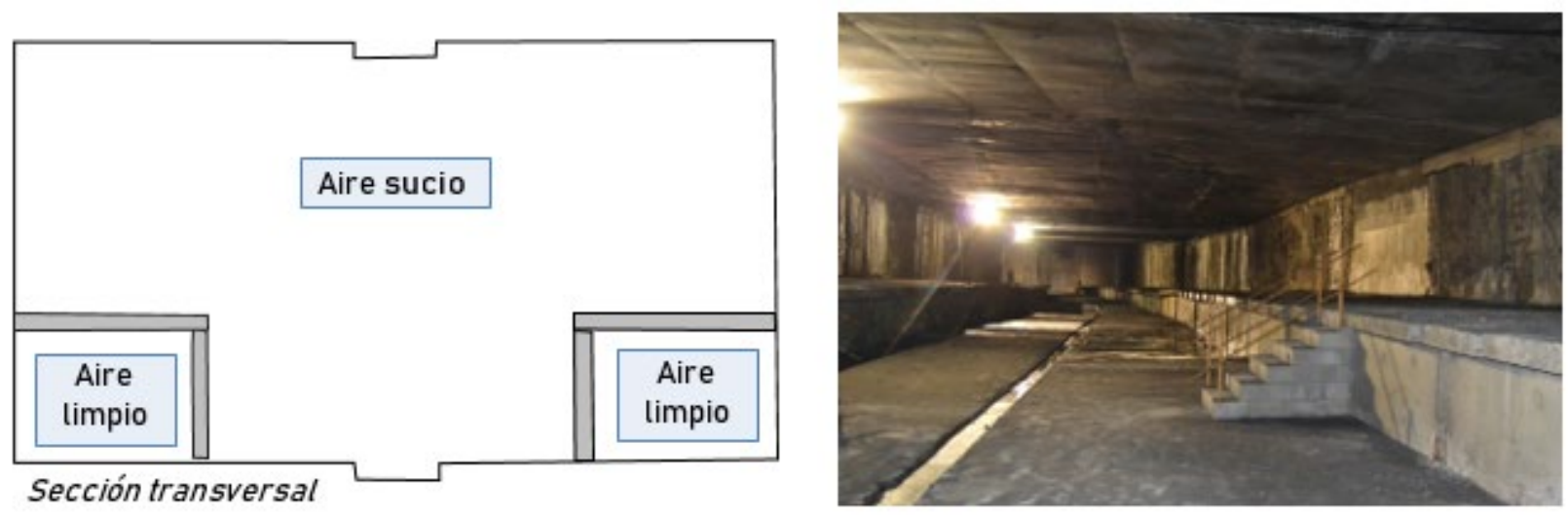

Figura 2. Sección transversal de la galería de ventilación (izquierda) e imagen tomada en el interior de la galería (derecha).

Dado que esta zona de la estructura se encuentra sometida a unas elevadas concentraciones de gases contaminantes, pues en ella se concentran los productos de las emisiones de los vehículos que circulan por esta zona de los túneles, se ha seleccionado como zona de estudio.

En este contexto, dado que se trabaja en una gran infraestructura urbana como Calle 30, es de gran importancia poder determinar y predecir el estado en el que se encuentra el hormigón que conforma el túnel. Cabe destacar que la corrosión de las armaduras del hormigón es un deterioro que implica, además de una posible reducción de la capacidad resistente de la estructura (Papadakis et al., 1989), un gran impacto económico, cuyos costes directos representan del orden del 3,1\% en países como Estados Unidos (Angst, 2018). Por ello, es necesario poder conocer y predecir tanto el alcance como el avance de esta patología para poder realizar actuaciones preventivas de mantenimiento o reparación antes de que su evolución sea desfavorable.

Por tanto, poder conocer el estado actual, pero también el estado futuro del deterioro del material supone un gran avance de cara a la planificación del mantenimiento y reparaciones sin necesidad de producir alteraciones en la circulación del tráfico. Para ello, se está desarrollando un modelo numérico que permita determinar el avance del frente de carbonatación en el hormigón, de manera que sea posible predecir el momento en el que esa disminución de $\mathrm{pH}$ en el interior del hormigón podría alcanzar a las armaduras y posibilitar su corrosión. Asimismo, este modelo se está calibrando con una campaña experimental llevada a cabo en laboratorio y en la propia galería de ventilación del túnel. 


\section{SIMULACIÓN NUMÉRICA DE LA CARBONATACIÓN}

Como se ha indicado anteriormente, en el proceso de la carbonatación intervienen una importante cantidad de parámetros que presentan una gran interdependencia, lo que hace que se trate de un fenómeno complejo y que su simulación sea complicada.

Existen en la actualidad una amplia variedad de modelos que estudian este fenómeno desde diferentes aproximaciones, desde modelos empíricos (Papadakis et al., 1991a; Parrott, 1994), estadísticos (Ann et al., 2010; Hills et al., 2015) o incluso de simulación mediante redes neuronales (Kwon and Song, 2010; Luo et al., 2014). Entre todos estos modelos, por su mayor precisión a la hora de analizar el proceso de deterioro del material, se ha seleccionado un modelo numérico, concretamente el modelo de Phung (Phung et al., 2016). Este modelo se desarrolla en el contexto del estudio de estructuras enterradas para eliminación de residuos radiactivos, sometidas a una elevada presión hidrostática y a muy altas concentraciones de $\mathrm{CO}_{2}$. Si bien se trata de un escenario diferente al del estudio de la carbonatación en túneles urbanos, este modelo es muy completo y detallado, por lo que se está procediendo a su adaptación y particularización a los condicionantes concretos de un túnel urbano.

El modelo de Phung (Phung et al. 2016) es un modelo unidimensional que tiene en cuenta las siguientes consideraciones: estudia tanto el transporte por difusión como el transporte por advección, tiene en cuenta la carbonatación de la portlandita y del gel CSH y asume que la hidratación del hormigón no varía durante el proceso de carbonatación.

Mediante estos supuestos, predice cambios en el frente de carbonatación, contenido de portlandita, variación del perfil de $\mathrm{pH}$ en el interior de la pieza, cantidad de $\mathrm{CO}_{2}$ absorbido y cambios en permeabilidad, porosidad, saturación y coeficiente de difusión.

Las principales modificaciones que deben realizarse para adaptarlo a los condicionantes propios de un túnel son las siguientes:

- Dado que el modelo de referencia está calibrado para pastas de cemento, debe realizarse su adaptación para poder trabajar con morteros y hormigones.

- El modelo considera el empleo de cemento tipo I y adiciones de filler calizo, por lo que debe adaptarse también para trabajar con adiciones de humo de sílice.

- Dado que el modelo existente está calibrado para simular ensayos a muy altas concentraciones de $\mathrm{CO}_{2}$ y elevadas presiones, debe adaptarse a los condicionantes de presión atmosférica y concentraciones de $\mathrm{CO}_{2}$ similares a las existentes en los túneles urbanos.

- El modelo base considera que la hidratación del hormigón es constante durante el proceso de carbonatación porque trabaja con ensayos cortos. Sin embargo, dado que se va a simular un proceso de carbonatación del orden de años, sí debe tenerse en cuenta esta variación de la hidratación.

El modelo se basa, fundamentalmente, en tres ecuaciones de conservación de la masa de aquellos agentes implicados en el proceso de carbonatación como son: el $\mathrm{CO}_{2}$ (en fase gaseosa y acuosa), el ion $\mathrm{Ca}^{2+}$ y el agua (en fase líquida y en fase vapor). 
Estas ecuaciones de balance de masa tienen la forma que se indica en la ecuación (2), en la que C es la concentración en el interior de la muestra $\left[\mathrm{kg} / \mathrm{m}^{3}\right]$, J es el flujo que la atraviesa $\left[\mathrm{kg} / \mathrm{m}^{2} \cdot \mathrm{s}\right]$ y Q es el término de reacción química $\left[\mathrm{kg} / \mathrm{m}^{3} \cdot \mathrm{s}\right]$.

$$
\frac{\partial C}{\partial t}+\frac{\partial J}{\partial x}=-Q
$$

En la Figura 3 se esquematiza el sentido físico de esta ecuación de conservación de masa. En dicha figura se representa una muestra de hormigón que tiene una cierta concentración en su interior $\mathrm{C}$, es atravesada por un flujo J y tiene una reactividad química Q. Esta ecuación indica que la variación de masa en el interior de la pieza con respecto al flujo que la atraviesa es constante. De esta manera, se combinan los conceptos de transporte con los de reactividad química, lo cual es de gran relevancia, dado que en el fenómeno de la carbonatación intervienen ambos procesos.

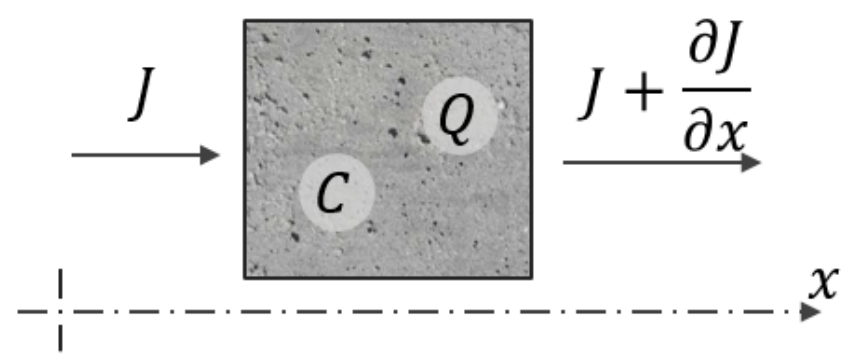

Figura 3. Esquema de funcionamiento de la ecuación de balance de masa en una muestra de hormigón atravesada por un flujo J.

Siguiendo el esquema de la ecuación (2), las ecuaciones diferenciales que describen el modelo se construyen como se indica a continuación. Tomando como ejemplo la ecuación de conservación del $\mathrm{CO}_{2}$, que se indica de manera simplificada en (3), se aprecia que tiene tres términos que se corresponden con la variación de la concentración en el interior de la pieza con el tiempo, la variación del flujo que la atraviesa y un término de reacción química.

$\frac{\partial\left(\emptyset(1-S) c_{g}+\emptyset S c_{w}\right)}{\partial t}-\frac{\partial\left(J_{g}+J_{w}\right)}{\partial x}=-r \emptyset M_{C O_{2}}$

El término de concentración en el interior de la pieza se divide a su vez en dos sumandos, que se corresponden con la concentración gaseosa $c_{\mathrm{g}}\left[\mathrm{kg} / \mathrm{m}^{3}\right]$ y la concentración acuosa $\mathrm{c}_{\mathrm{w}}\left[\mathrm{kg} / \mathrm{m}^{3}\right]$ existentes en el interior de los poros, donde $\mathrm{S}$ es la saturación [-] y $\Phi$ es la porosidad [-]. Ambas concentraciones se relacionan mediante la Ley de Henry.

El término correspondiente a la variación del flujo tiene en cuenta también las fases gaseosa y acuosa, siendo $J_{\mathrm{g}}$ el flujo de la fase gaseosa $\left[\mathrm{kg} / \mathrm{m}^{2} \cdot \mathrm{s}\right]$ y $J_{\mathrm{w}}$ el flujo de la fase acuosa $\left[\mathrm{kg} / \mathrm{m}^{2} \cdot \mathrm{s}\right]$. Estos flujos, a su vez, se descomponen en dos términos dado que tienen en cuenta tanto el flujo por advección como el flujo por difusión. En (4) se indica la ecuación del flujo gaseoso $\mathrm{J}_{\mathrm{g}}$, siendo la del flujo acuoso $\mathrm{J}_{\mathrm{w}}$ una expresión análoga.

$J_{g}=-\left[c_{g} v_{g}+D_{e f, g} \frac{\partial c_{g}}{\partial x}\right]$ 
El primer término de la ecuación (4) se corresponde con el transporte por advección, en el que $\mathrm{vg}$ es la densidad de flujo volumétrico de la fase gaseosa $[\mathrm{m} / \mathrm{s}$ ] que se calcula mediante la Ley de Darcy. El segundo término de la ecuación se corresponde con el transporte por difusión que se calcula mediante la Ley de Fick, donde $\mathrm{D}_{\text {ef,g }}$ es el coeficiente de difusión efectivo de la fase gaseosa $\left[\mathrm{m}^{2} / \mathrm{s}\right]$.

En cuanto al último término, el correspondiente a la reactividad química, tiene en cuenta tanto la variación de concentración debida a la portlandita como la correspondiente al CSH, como se indica en (5).

$r=-\frac{\partial[\mathrm{CH}]}{\partial t}-\frac{\partial[\mathrm{CSH}]}{\partial t}$

Con este modelo, que se está implementando, adaptando y calibrando mediante el software COMSOL Multiphysics, se han obtenido ciertos resultados preliminares que se muestran seguidamente. Actualmente se está trabajando con una geometría como la representada en la Figura 4, que se trata de una pieza de hormigón unidimensional de $3 \mathrm{~cm}$ que se encuentra sometida a la acción del $\mathrm{CO}_{2}$ en su extremo izquierdo.

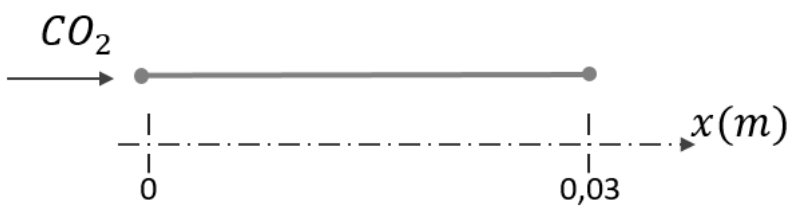

Figura 4. Esquema de la pieza unidimensional de hormigón que se emplea en el modelo.

Se ha comprobado, como se muestra en la Figura 5, que a medida que aumenta la porosidad de la muestra de hormigón (a igualdad del resto de factores, tanto de composición como de exposición), aumenta también la concentración de $\mathrm{CO}_{2}$ en la fase acuosa de los poros. Este resultado se debe a que el avance de la carbonatación es más notable en aquellos hormigones que presentan una mayor porosidad (Valcuende and Parra, 2010).

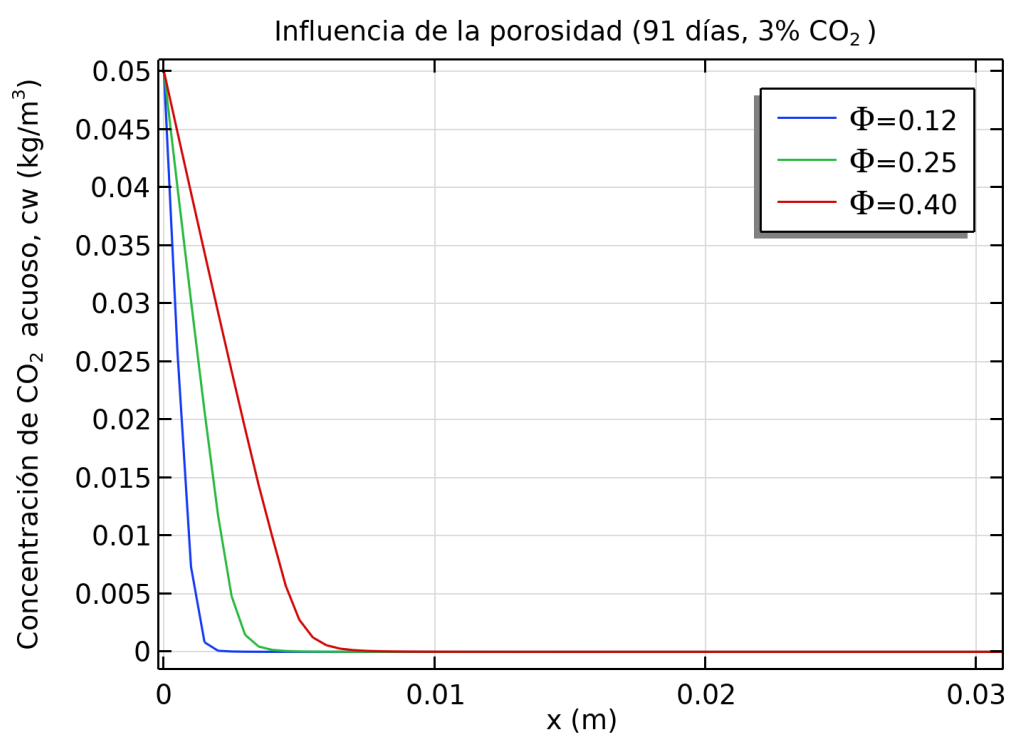

Figura 5. Influencia de la variación de porosidad en el avance de la concentración de $\mathrm{CO}_{2}$ acuoso en el interior de la pieza de hormigón. 
Además, la carbonatación tiene un efecto reductor de la porosidad en la zona carbonatada con respecto a la zona sana del hormigón, dado que, por un lado, el $\mathrm{CaCO}_{3}$ que se forma tiene un volumen mayor que la portlandita que desaparece; y, por otro, aunque la descalcificación del CSH que podría aumentar en cierta medida la porosidad de la muestra, al formarse también $\mathrm{CaCO}_{3}$ a partir del calcio del CSH, la porosidad total de la muestra disminuye (Morandeau, 2014). Este comportamiento se aprecia en la Figura 6, donde se observa cómo a medida que aumenta el tiempo de exposición de la muestra al ambiente de carbonatación, la porosidad de la zona carbonatada disminuye.

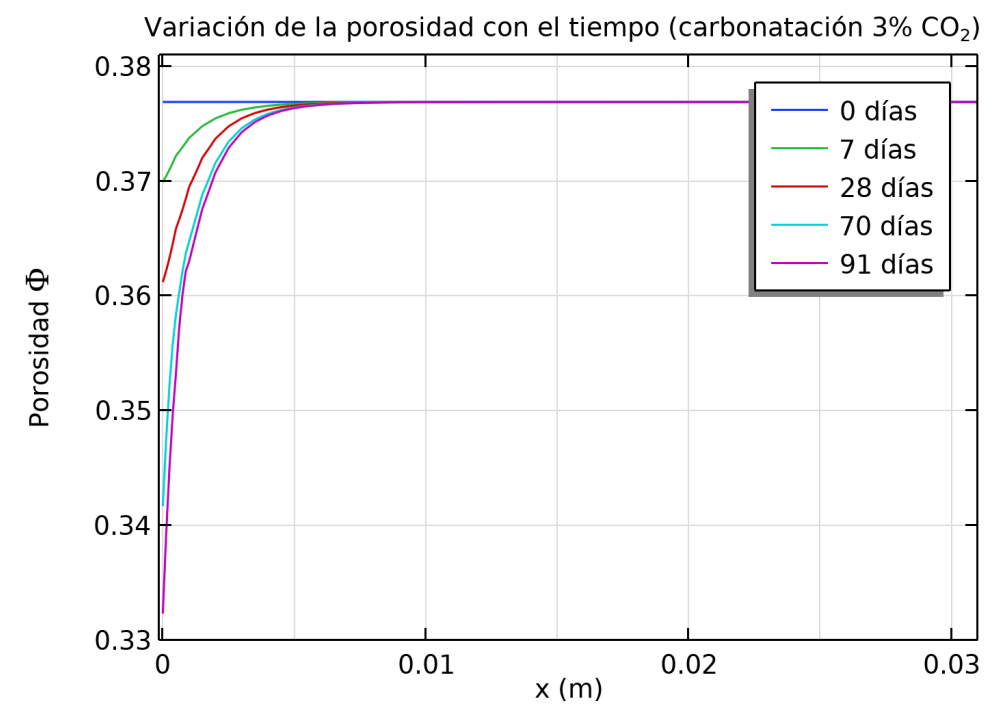

Figura 6. Variación de la porosidad en la zona carbonatada con el tiempo de exposición de la pieza al $\mathrm{CO}_{2}$.

En cuanto al efecto de la saturación en la porosidad, es conocido que valores muy bajos ralentizan la carbonatación (dado que para que se produzca el deterioro es necesario contar con la presencia de humedad) y que valores muy altos de saturación son contrarios también al avance de la carbonatación, dado que dificulta la entrada del $\mathrm{CO}_{2}$ gaseoso en el poro (Papadakis et al., 1991b). Este último comportamiento se muestra en la Figura 7, donde se observa que, en rangos altos de saturación, a medida que aumenta este parámetro (a igualdad del resto de factores, tanto de composición como de exposición), disminuye también la concentración de $\mathrm{CO}_{2}$ en la fase acuosa de los poros. 


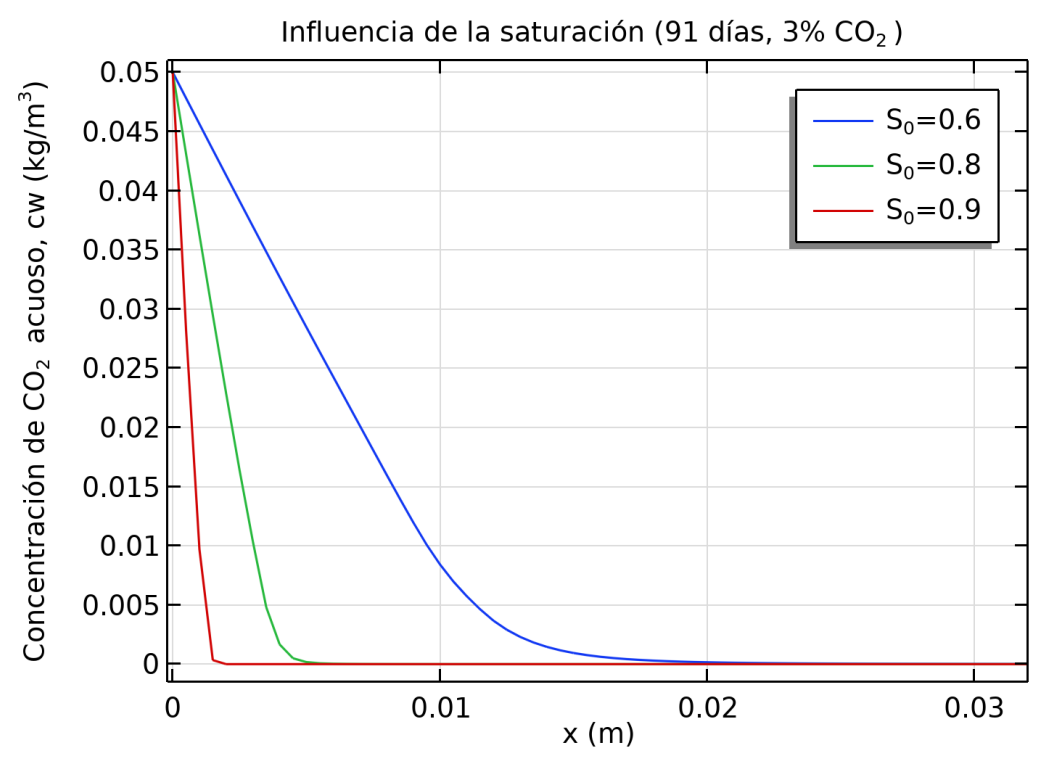

Figura 7. Influencia de la variación del grado de saturación en el avance de la concentración de $\mathrm{CO}_{2}$ acuoso en el interior de la pieza de hormigón.

\section{CAMPAÑA EXPERIMENTAL}

Para poder calibrar el modelo anteriormente descrito y adaptarlo a las condiciones particulares del túnel de estudio, se está llevando a cabo una campaña experimental en dos fases. Por un lado, una fase realizada en laboratorio mediante la fabricación de morteros y hormigones; y otra fase diferenciada, en la que se analizan muestras y se estudian datos tomados en el interior del túnel.

En la campaña experimental que se está llevando a cabo en el laboratorio, se han fabricado morteros y hormigones con diferentes dosificaciones variando la relación agua/cemento y empleando cemento tipo CEM I con y sin adiciones de humo de sílice en unos porcentajes de $6 \%$ y $10 \%$ con respecto al peso de cemento. Se seleccionaron estas adiciones debido a que, según el Pliego de Prescripciones Técnicas del Proyecto de Construcción, los hormigones empleados en la construcción de los túneles tenían una cierta adición de humo de sílice.

Sobre estas muestras, se están llevando a cabo ensayos de caracterización del material y ensayos de carbonatación acelerada y natural, como los que se muestran en la Figura 8. En la imagen de la izquierda se muestra una probeta de mortero sobre la que se han pulverizado tres indicadores distintos: fenolftaleína $(8,2-9,8)$, timolftaleína $(8,6-10,0)$ y rojo de cresol $(7,0-8,8)$. Dado que estos tres indicadores viran a diferentes intervalos de $\mathrm{pH}$, se obtiene una mayor información de este ensayo. Como se observa en la imagen, el mayor frente se obtiene con la timolftaleína, seguido de la fenolftaleína y del rojo de cresol. En cuanto a este último indicador, se ha seleccionado por su bajo $\mathrm{pH}$ de viraje, dado que está previsto realizar también ensayos acelerados con $\mathrm{SO}_{2}$ que, previsiblemente, producirán un mayor descenso del $\mathrm{pH}$ de las muestras. 

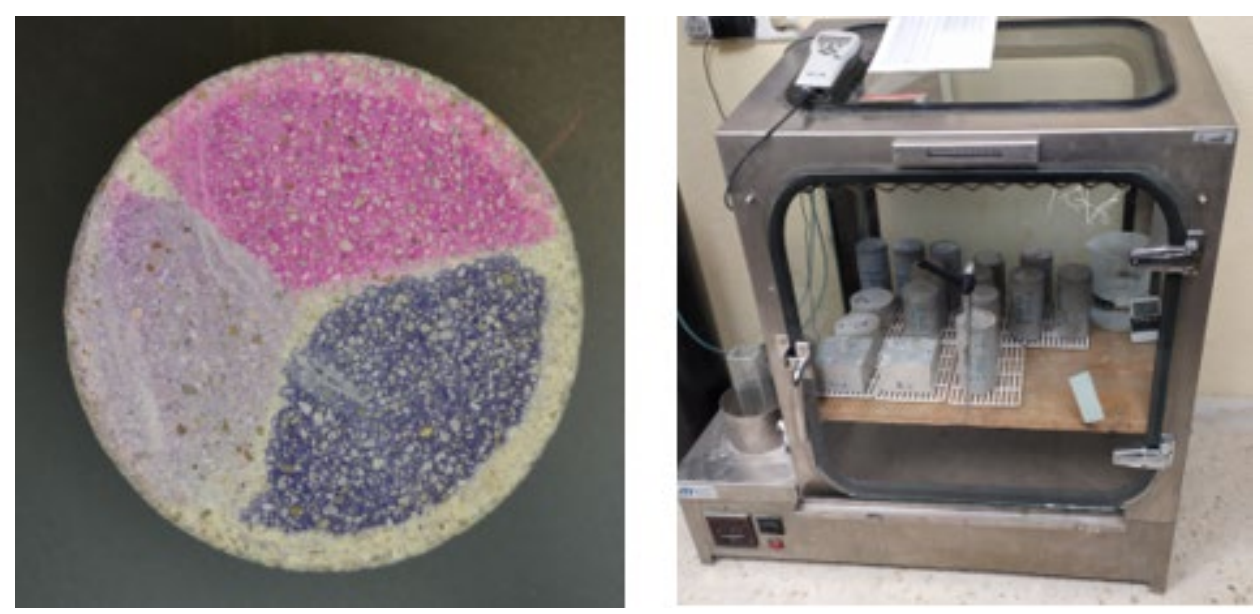

Figura 8. Ensayos de carbonatación llevados a cabo en morteros. Empleo de tres indicadores (izquierda) y cámara de carbonatación acelerada (derecha).

En cuanto a la campaña experimental que se está llevando a cabo en el interior del túnel, se ha realizado una determinación de las concentraciones de gases existentes en el interior de la galería de ventilación. Esta toma de datos se realizó durante un periodo de 15 días y se midieron concentraciones de $\mathrm{CO}_{2}, \mathrm{CO}, \mathrm{SO}_{2}, \mathrm{NO}_{x}, \mathrm{H}_{2} \mathrm{~S}$ y $\mathrm{O}_{3}$ y de partículas PM 1, PM 2,5 y PM 10; así como parámetros ambientales. Las medias de los valores registrados se muestran en la Figura 9, donde se observa que el gas que presenta una mayor concentración es el $\mathrm{CO}_{2}$, seguido en menor medida por las concentraciones de $\mathrm{CO}, \mathrm{SO}_{2} \mathrm{y} \mathrm{NO}_{\mathrm{x}}$. Se han registrado también unas ciertas concentraciones de $\mathrm{H}_{2} \mathrm{~S}$ y $\mathrm{O}_{3}$.

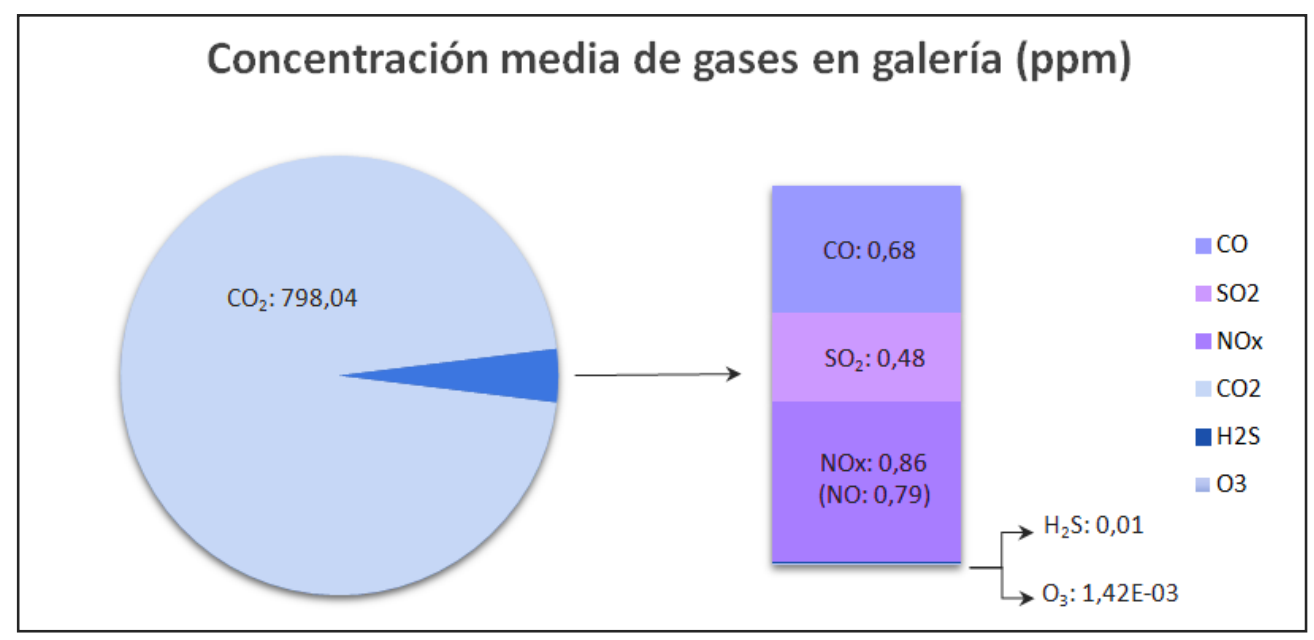

Figura 9. Ensayos de carbonatación llevados a cabo en morteros. Empleo de tres indicadores (izquierda) y cámara de carbonatación acelerada (derecha).

Mediante esta toma de datos, además de conocer las concentraciones de gases que existen en la galería de ventilación, se ha observado que su variación a lo largo del día y a lo largo de la semana se encuentra fuertemente influenciada por el tráfico que circula por la calzada (Figura 10). Para llegar a esta conclusión, se ha realizado también un estudio del tráfico que circuló durante los días de toma de datos por la calzada del túnel, así como de las concentraciones de $\mathrm{CO}$ y $\mathrm{NO}$ que se miden diariamente como parte de la gestión de la infraestructura. 


\section{Comparación de concentración de NO con intensidad de tráfico}

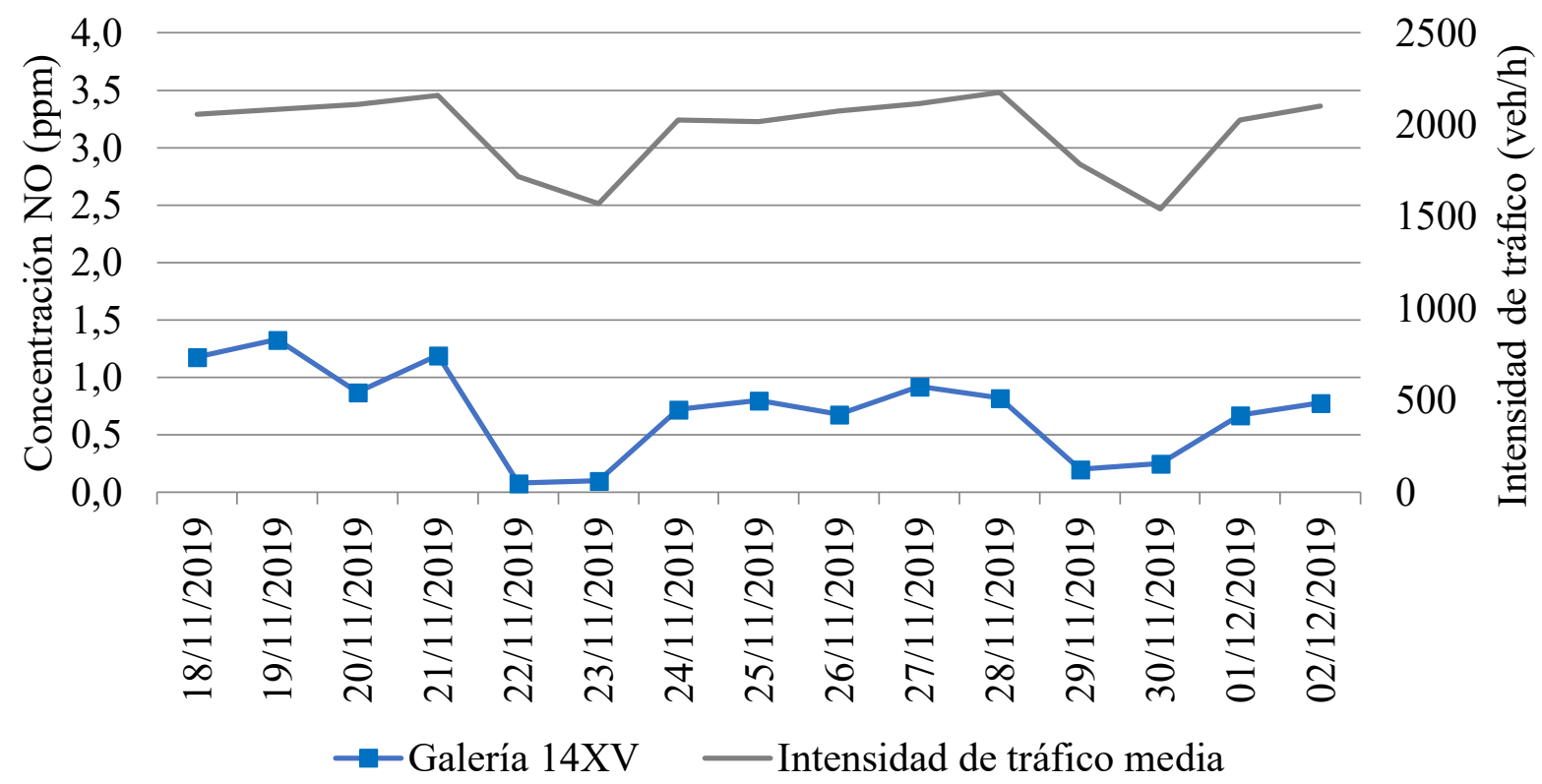

Figura 10. Comparación de la concentración de NO medida en la galería de ventilación y la intensidad de tráfico que circulaba por la calzada.

La importancia y el interés de esta conclusión residen en la capacidad de realizar una estimación precisa de la concentración de gases a la que se ha visto expuesto el hormigón de la galería desde su construcción, basada en el registro de intensidades de tráfico del que se dispone. De esta manera, y mediante la extracción y análisis de testigos del hormigón de la galería, es posible conocer el grado de deterioro del material que ha producido dicha concentración de gases. Pero, además, posibilita también realizar una predicción a futuro de las concentraciones a las que se verá expuesta la galería en los próximos años y, por tanto, estimar el grado de deterioro que presentará el material y la necesidad, o no, de reparación de la estructura.

\section{CONCLUSIONES}

En los túneles urbanos se puede producir el deterioro del hormigón que los conforma debido a la neutralización provocada por los gases de los vehículos que circulan por sus calzadas y, en especial, a la carbonatación que produce el $\mathrm{CO}_{2}$. Estos mecanismos de deterioro provocan un descenso del $\mathrm{pH}$ en el interior del material (de 12,6 en hormigón sano a 8,3) que puede comprometer la durabilidad del acero de las armaduras, dado que su despasivación se produce cuando se encuentran en un entorno con un $\mathrm{pH}$ inferior a 9, favoreciendo la aparición de la corrosión.

Es de gran importancia poder conocer y predecir el estado de deterioro de una infraestructura de gran envergadura como los túneles de la Calle 30 en Madrid, dado que la corrosión implica consecuencias tanto estructurales como económicas.

La carbonatación del hormigón es un fenómeno complejo y su simulación no es sencilla, por lo que para poder predecir y conocer el estado del material, es importante poder contar con modelos de deterioro y ciclo de vida de la estructura. Estos modelos deben calibrarse mediante una campaña experimental.

El empleo de estos modelos como herramienta para la conservación de estructuras es de gran relevancia, dado que permiten conocer y predecir el estado de deterioro del material sin que se afecte al tráfico que circula por las mismas. 


\section{AGRADECIMIENTOS}

Los autores agredecen al Ministerio de Ciencia e Innovación la concesión de la ayuda BIA201678742-C2-2-R, y a Calle 30 la financiación de la Cátedra Universidad Empresa “Calle 30".

\section{REFERENCIAS}

Angst, U. M. 2018. "Challenges and Opportunities in Corrosion of Steel in Concrete." Materials and Structures/Materiaux et Constructions 51(1):1-20. doi: 10.1617/s11527-017-1131-6.

Ann, K. Y., S. W. Pack, J. P. Hwang, H. W. Song, and S. H. Kim. 2010. "Service Life Prediction of a Concrete Bridge Structure Subjected to Carbonation." Construction and Building Materials 24(8):1494-1501. doi: 10.1016/j.conbuildmat.2010.01.023.

Galán, I. 2011. “Carbonatación Del Hormigón: Combinación de CO2 Con Las Fases Hidratadas Del Cemento y Frente de Cambio de PH." Universidad Complutense de Madrid.

Galan, I., Balderman A., Kusterle, W., Dietzel, M. and Mittermayr, F. 2019. "Durability of Shotcrete for Underground Support- Review and Update." Construction and Building Materials 202:465-93. doi: 10.1016/j.conbuildmat.2018.12.151.

Hills, T. P., Gordon, F., Florin, N. H. and Fennell, P. S. 2015. "Statistical Analysis of the Carbonation Rate of Concrete." Cement and Concrete Research 72:98-107. doi: 10.1016/j.cemconres.2015.02.007.

Tongaria K., Mandal S. and Mohan, D. 2018. "A Review on Carbonation of Concrete and Its Prediction Modelling." Journal of Environmental Nanotechnology 7(4):75-90. doi: 10.13074/jent.2018.12.184325.

Kwon, S J and Song, H.W. 2010. "Analysis of Carbonation Behavior in Concrete Using Neural Network Algorithm and Carbonation Modeling." Cement and Concrete Research 40(1):11927. doi: 10.1016/j.cemconres.2009.08.022.

Li, C., Wu, M., Chen, Q. and Jiang, Z. 2018. "Chemical and Mineralogical Alterations of Concrete Subjected to Chemical Attacks in Complex Underground Tunnel Environments during 20-36 Years." Cement and Concrete Composites 86:139-59. doi: 10.1016/j.cemconcomp.2017.11.007.

Llopis Serrano, G. 2012. "Inspección de Túneles de Carretera de La Dirección General de Carreteras Del Ministerio de Fomento." Rutas 149:33-38.

López-de Abajo, L., Alberti, M.G. and Gálvez, J. C. 2020. "Critical Review on Concrete Carbonation: Factors Involved and Mathematical Modelling." in V International Conference on Technological Innovation in Building. Madrid.

López-de Abajo, L, Pérez-Fortes, A. P, Alberti, M.G., Gálvez, J.C. and Ripa, T. 2020. "Sustainability Analysis of the M-30 Madrid Tunnels and Madrid Río after 14 Years of Service Life." Applied Sciences (Switzerland) 10(20):1-20. doi: 10.3390/app10207368.

Luo, D., Niu, D. and Dong, Z. 2014. "Application of Neural Network for Concrete Carbonation Depth Prediction." Proceedings of the 4th International Conference on the Durability of Concrete Structures, ICDCS 2014 66-71. doi: 10.5703/1288284315384.

Ministerio de Fomento. 2010. Instrucción de Hormigón Estructural. EHE-08. 4th ed. Ministerio de Fomento.

Ministerio de Fomento. 2012. Guía Para La Realización de Inspecciones Principales de Obras de Paso En La Red de Carreteras Del Estado.

Morandeau, A., Thiéry, M. and Dangla, P. 2014. "Investigation of the Carbonation Mechanism of $\mathrm{CH}$ and C-S-H in Terms of Kinetics, Microstructure Changes and Moisture Properties." Cement and Concrete Research 56:153-70. doi: 10.1016/j.cemconres.2013.11.015. 
Núñez-Alonso, D, Pérez-Arribas, L.V., Manzoor, S. and J. O. Cáceres, J. O.. 2019. “Statistical Tools for Air Pollution Assessment: Multivariate and Spatial Analysis Studies in the Madrid Region." Journal of Analytical Methods in Chemistry 2019. doi: 10.1155/2019/9753927.

Papadakis, V. G., Vayenas, C.G. and Fardis, M. N. 1989. "A Reaction Engineering Approach to the Problem of Concrete Carbonation." AIChE Journal 35(10):1639-50. doi: 10.1002/aic.690351008.

Papadakis, V. G., Vayenas, C.G. and Fardis, M. N. 1991a. "Experimental Investigation and Mathematical Modeling of the Concrete Carbonation Problem." Chemical Engineering Science 46(5-6):1333-38. doi: 10.1016/0009-2509(91)85060-B.

Papadakis, V. G., Vayenas, C.G. and Fardis, M. N. 1991b. "Physical and Chemical Characteristics Affecting the Durability of Concrete." ACI Materials Journal 88(2):186-96. doi: 10.14359/1993.

Parrott, L. J. 1994. "Design for Avoiding Damage Due to Carbonation-induced Corrosion." in CANMET/ACI Conference on Durability of Concrete. Nice, France.

Perez-Prada, F., and Monzon, A. 2014. "How Lowering Speed Limits in an Urban Highway Affects Traffic Perfirmance and Emissions: The Caso of Madrid M-30 Ring-Road." Pp. 1-16 in XI Congreso de Ingeniería del Transporte, CIT 2014. Santander, Spain.

Perez-Prada, F, and Monzon, A. 2017. "Ex-Post Environmental and Traffic Assessment of a Speed Reduction Strategy in Madrid's Inner Ring-Road." Journal of Transport Geography 58:25668. doi: 10.1016/j.jtrangeo.2016.12.018.

Phung, Q.T., Maes, N., Jacques, D., De Schutter, G., Ye, G. and Perko, J. 2016. "Modelling the Carbonation of Cement Pastes under a CO2 Pressure Gradient Considering Both Diffusive and Convective Transport." Construction and Building Materials 114:333-51. doi: 10.1016/j.conbuildmat.2016.03.191.

Richardson, M. 2002. Fundamentals of Durable Reinforced Concrete. First ed. London: Spon Press.

Singh, N. and Singh, S. P.. 2016. "Reviewing the Carbonation Resistance of Concrete." Journal of Materials and Engineering Structures 3:35-57.

Valcuende, M., and Parra, C. 2010. "Natural Carbonation of Self-Compacting Concretes." Construction and Building Materials 24(5):848-53. doi: 10.1016/j.conbuildmat.2009.10.021. 